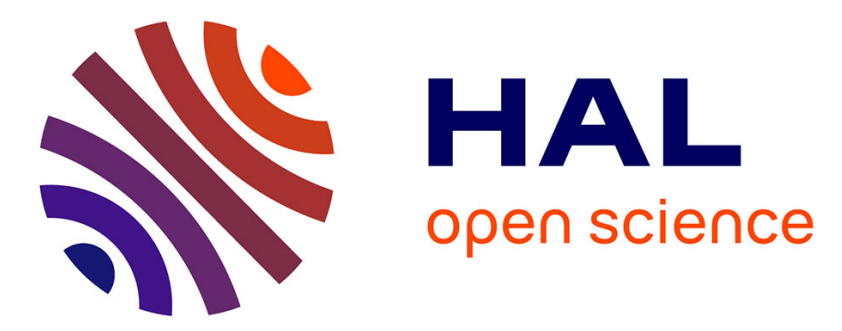

\title{
Coherence between the excitation of states with different energies
}

\author{
J. de Gouw, J. van Eck, S. van Der Aart, H. Heideman
}

\section{To cite this version:}

J. de Gouw, J. van Eck, S. van Der Aart, H. Heideman. Coherence between the excitation of states with different energies. Journal de Physique IV Proceedings, 1993, 03 (C6), pp.C6-207-C6216. 10.1051/jp4:1993620 . jpa-00251705

\section{HAL Id: jpa-00251705 https://hal.science/jpa-00251705}

Submitted on 1 Jan 1993

HAL is a multi-disciplinary open access archive for the deposit and dissemination of scientific research documents, whether they are published or not. The documents may come from teaching and research institutions in France or abroad, or from public or private research centers.
L'archive ouverte pluridisciplinaire HAL, est destinée au dépôt et à la diffusion de documents scientifiques de niveau recherche, publiés ou non, émanant des établissements d'enseignement et de recherche français ou étrangers, des laboratoires publics ou privés. 


\title{
Coherence between the excitation of states with different energies
}

\author{
J.A. DE GOUW, J. VAN ECK, S. VAN DER AART and H.G.M. HEIDEMAN \\ Debye Institute, Department of Atomic and Interface Physics, Utrecht University, P.O. Box 80000, \\ 3508 TA Utrecht, The Netherlands
}

\begin{abstract}
In recent experiments we have shown both in electron impact and in photoionisation studies the possibility to determine coherences between the excitation of states of different energies. In this paper the consequences of applying coincident techniques to these experiments are discussed. In a coincidence experiment one has to choose, however, for lower energy resolutions in order to reach sufficient count rates. We present an experiment which shows that these lower resolutions do not totally obscure the formerly observed interference effects, thus proving the feasibility of a future coincidence experiment.
\end{abstract}

\section{Introduction}

During the past two decades important information on atomic collision processes has been obtained from the observation of coherences between the excitation of different atomic states. Such information provides crucial tests for theoretical models on the most fundamental level. According to the quantum dynamical picture of an atomic collision process the final state wavefunction $\mid \Phi>$ can be represented as a coherent superposition of the possible final states $\left|a_{n}\right\rangle$ :

$$
\left|\Phi>=\sum_{n} c_{n}\right| a_{n}>,
$$

in which the summation may also include an integration over continuum states. The excitation amplitudes $c_{n}$ are in general complex numbers. Their absolute values squared represent the excitation cross sections for the different final states, whereas their relative phases represent the coherences between the excitation of the various states. A measurement of the coherence between the excitation of two states $\left|a_{n}\right\rangle$ and $\left|a_{m}\right\rangle$ involves an analysis of the interference between the excitation of these states, which is determined by terms of the type $c_{n} c_{m}^{*}$.

Up until now most coherence measurements have been performed on degenerate magnetic substates. These measurements are performed using electron-photon or electron-electron coincidence techniques [1]. Coherences between states of different energies are in general not easy to observe. For small energy differences they may be observable as quantum beats [2]. For larger energy differences interferences and hence coherences can only be observed when the different states can decay to final states that are indistinguishable. Such a situation occurs, for instance, in the near-threshold, electron-impact excitation and subsequent decay of autoionising states. In this case the energy distributions of scattered and ejected electrons from different autoionising states can be made to overlap due to a broadening and an energy shift of these distributions as a result of a post-collision interaction $[3][4][5]$. 
In this paper we will discuss two experiments in which we have demonstrated the possibility to observe coherences between the excitation of states of different energies without employing shifting and broadening effects such as those caused by post-collision interaction. One is an electron-impact experiment of van den Brink et al performed some years ago by our group, the other is a recent photoionisation experiment also by our group. Both experiments are non-coincidence experiments, based on the observation of interference between primary and secondary emitted electrons. The consequences of applying coincidence techniques to these experiments will be discussed and some interesting conclusions will be made. To actually do a coincidence experiment one has to choose for lower energy resolutions in order to reach sufficient count rates. The question arises whether this limited resolution obscures the formerly observed interference or not. We will present an experiment which shows that despite a limited resolution the interference can still be observed.

\section{Coherence in autoionisation processes}

Van den Brink et al [6] have been able for the first time to observe coherences between autoionising states of different energies without employing shifting and broadening effects such as those caused by post-collision interaction. Their method was based on the observation of interferences between scattered and ejected electrons, resulting from the electron impact excitation and subsequent decay of different autoionising states of helium. Consider the following two processes:

$$
\begin{aligned}
& e+\mathrm{He} \rightarrow \mathrm{He}^{* *}(1)+e_{s}^{(1)} \rightarrow \mathrm{He}^{+}+e_{j}^{(1)}+e_{s}^{(1)} \\
& e+\mathrm{He} \rightarrow \mathrm{He}^{* *}(2)+e_{s}^{(2)} \rightarrow \mathrm{He}^{+}+e_{j}^{(2)}+e_{s}^{(2)},
\end{aligned}
$$

in which $e_{s}$ represents a scattered and $e_{j}$ an ejected electron. The energy of the incident electrons can be chosen such that the energy of $e_{s}^{(1)}$ equals the energy of $e_{j}^{(2)}$, and vice versa. In that case the final states of both processes can not be distinguished and interferences should occur. This physical situation is completely analogous to Young's classical two-slit experiment.

The experiment of van den Brink et al was done using an energy-selected electron beam with a small energy width of $65 \mathrm{meV}$ and a hemispherical analyser to measure the electrons. Spectra were taken in the so-called "constant energy loss" mode, by varying both the incident electron energy and the detection energy, keeping the difference between them constant. The ejected electrons from an autoionising state always appear at the same detection energy. The constant energy loss can be chosen equal to the excitation energy of an autoionising state, in which case the scattered electrons from that state will appear as an interfering "background" in the spectra. One of the most striking results of van den Brink et al is shown in figure 1. The spectra show the ejected-electron peaks from the $\left(2 s^{2}\right)^{1} S,(2 s 2 p)^{3} P,\left(2 p^{2}\right)^{1} D$ and $(2 s 2 p)^{1} P$ autoionising states of helium. The energy loss is varied stepwise through the excitation energy of the $(2 s 2 p)^{1} P$ state and in the appropriate spectra the "background" contains therefore the scattered electrons from this state. The interference between scattered and ejected electrons is clearly visible, the most striking feature being the strong increase of the $(2 s 2 p)^{3} P$ peak and the complete disappearance of the $\left(2 s^{2}\right)^{1} S$ structure when the energy loss approaches the $(2 s 2 p)^{\mathbf{1} P}$ excitation energy.

\section{Coherence in inner-shell photoionisation}

In a recent experiment [7] we applied the method of van den Brink et al to study coherences between the photoionisation of different inner-shell vacancy states of argon, lying as far as $75 \mathrm{eV}$ apart. Here, the different vacancy states can decay to the same final state through the Auger channel. Consider, again, two processes:

$$
\begin{aligned}
& \mathrm{h} \nu+\mathrm{Ar} \rightarrow \mathrm{Ar}^{+} 2 s^{-1}+e_{p h}^{(1)} \rightarrow \mathrm{Ar}^{2+}+e_{A}^{(1)}+e_{p h}^{(1)} \\
& \mathrm{h} \nu+\mathrm{Ar} \rightarrow \mathrm{Ar}^{+} 2 p^{-1}+e_{p h}^{(2)} \rightarrow \mathrm{Ar}^{2+}+e_{A}^{(2)}+e_{p h}^{(2)},
\end{aligned}
$$




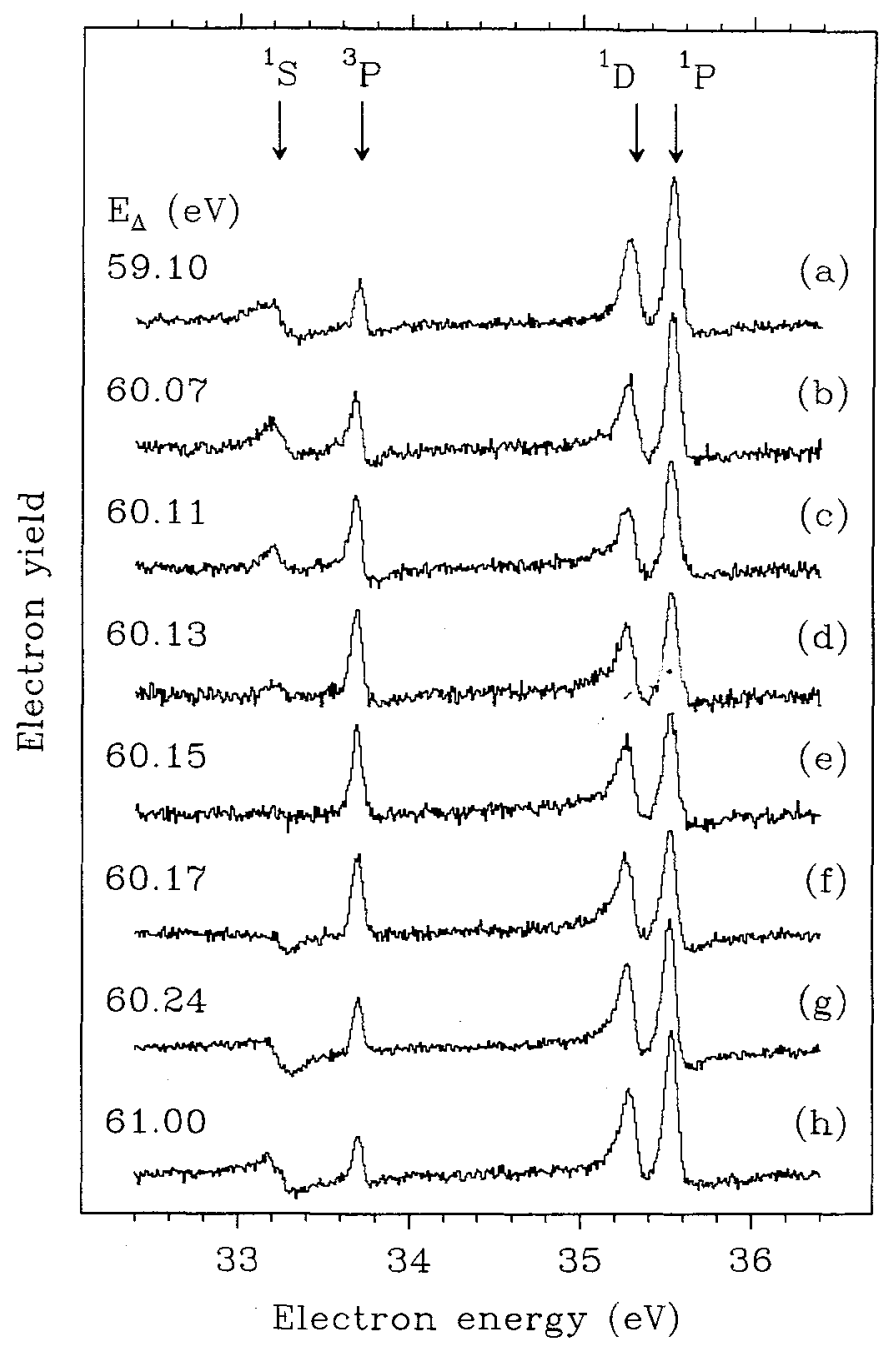

Figure 1: Ejected-electron peaks from the $\left(2 s^{2}\right)^{1} S,(2 s 2 p)^{3} P,\left(2 p^{2}\right)^{1} D$ and $(2 s 2 p)^{1} P$ autoionising states of helium. The spectra are measured in the "constant energy loss" mode. The energy loss is varied stepwise through the excitation energy of the $(2 s 2 p)^{1} P$ state and the "background" contains therefore in the appropriate spectra scattered electrons from this state. Figure is taken from van den Brink et al [8]. 
in which $e_{p h}$ represents a photoelectron and $e_{A}$ an Auger electron. By adjusting the photon energy such that the energy of the photoelectron $\mathrm{e}_{p h}^{(1)}$ becomes equal to the energy of the Auger electron $e_{A}^{(2)}$ and vice versa, the final states of the two processes become indistinguishable and interferences should occur.

This experiment was done using synchrotron radiation as a photon source and a cylindrical mirror analyser to measure the electrons. The experimental method was slightly different from the method used in the measurements described in section 2. In the photoionisation experiment spectra were taken in the "constant detection energy" mode, by varying the photon energy at a constant detection energy. Photoelectrons appear in the spectra when the photon energy minus the ionisation threshold equals the detection energy. The detection energy can be chosen equal to one of the Auger energies, in which case the Auger electrons will appear as an interfering "background" in the spectra. The result of our measurements is shown in figure 2. The peak in figure $2 \mathrm{a}$ consists of $2 \mathrm{~s}$ photoelectrons. The solid line represents a least-squares fit of a Lorentzian line shape, which appears to describe the data well, as expected. The structure in figure $2 \mathrm{~b}$ consists of $2 s$ photoelectrons on an interfering background of $L_{3} M_{2,3} M_{2,3}\left({ }^{1} D_{2}\right)$ Auger electrons. The only difference between the two measurements was a small change in the detection energy. The photoelectron peak should have shifted by the same amount. The solid line in figure $2 \mathrm{~b}$ represents a fit of a Lorentzian line shape, which has been shifted in energy by precisely that amount. It is clear that the measured peak is shifted relative to the energy where it should be. Moreover, the measured line shape appears to be asymmetrical. We attribute the disagreement to the interference between Auger and photoelectrons. Van den Brink et al have derived a parametrised line shape that is able to account for this kind of interference effects [8]. Figure 2c shows the same result as figure $2 \mathrm{~b}$, but now the solid line represents a fit using this line shape. It is clear that the parametrisation of van den Brink et al is adequatly able to describe the observed line shape and we conclude, therefore, that we have observed the coherence between the excitation of two inner-shell vacancy states.

The system of photoionisation and subsequent Auger decay has several advantages compared to the work of van den Brink et al. Photoionisation is much better understood than the excitation of an autoionising state, due to the applicability of the dipole approximation and to the very limited number of angular momenta involved. Moreover, in photoionisation the contribution of the direct double ionisation channel is negligible, whereas in the experiment of van den Brink et al the direct ionisation channel is quite large. It should be possible, therefore, to develop a theory that can describe our present photoionisation experiment, whereas it will be very hard to construct a theory that can quantitatively explain the interference phenomena seen in the experiment of van den Brink et al.

\section{Coincidence experiments}

The two experiments described in the sections 2 and 3 are non-coincidence experiments, in which only one electron is detected, which is either the scattered or the ejected electron in the work of van den Brink et al, or either the Auger or the photoelectron in our present work. This implies that, in our case, the detection of photoelectrons (Auger electrons) is integrated over all directions of the non-observed Auger electrons (photoelectrons). Despite this integration the interference is still visible, which is a somewhat surprising finding.

In a coincidence experiment the integration over all directions of the non-observed electron is no longer necessary. The emission direction of the second electron can be determined as an extra parameter and it can be expected that much larger interference effects will be observed.

One has to keep in mind that the final states of the two interfering processes must remain indistinguishable. If the time resolution of the coincident measurement would be very high, this would no longer be true. The photoelectron arrives before the Auger electron at the detector, and the two electrons can therefore in principle be distinguished by their time order. The interferences would 


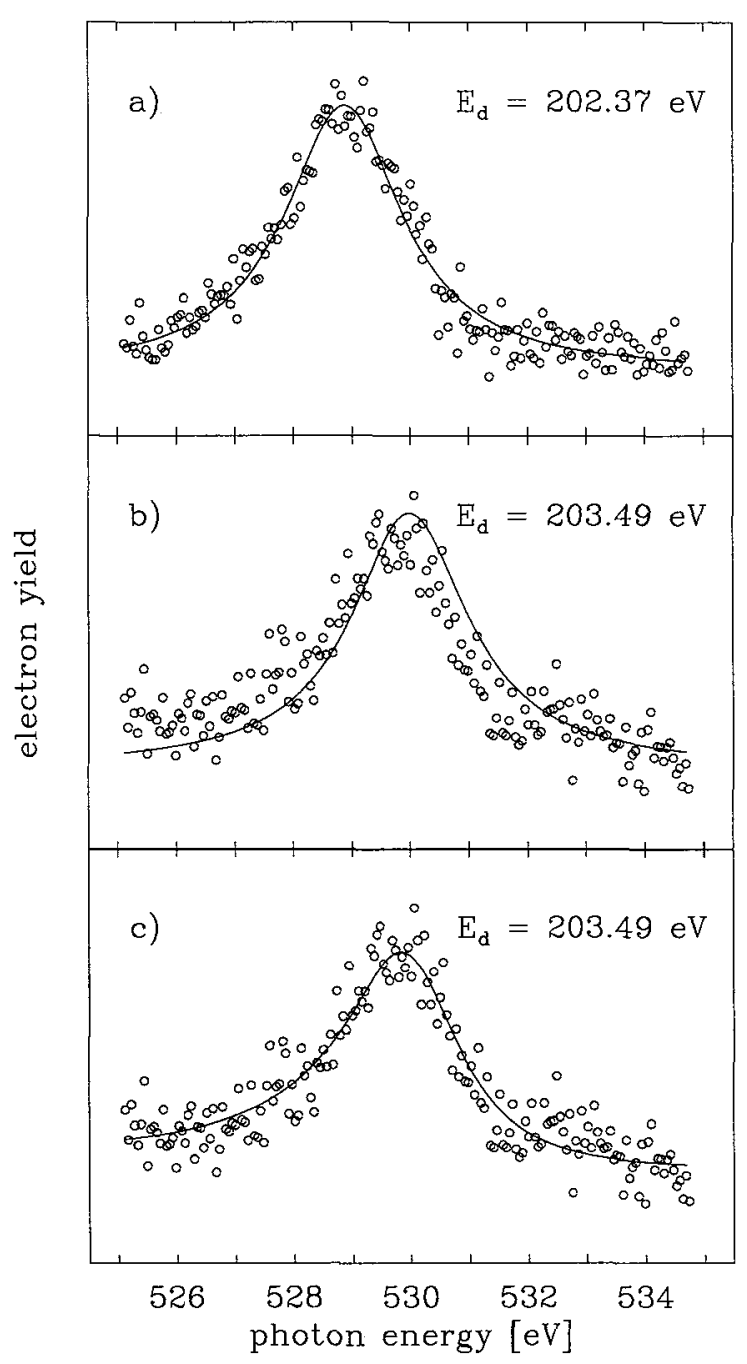

Figure 2: (a) Ar $2 \mathrm{~s}^{-1}$ photoelectrons $\left(\mathrm{e}_{p h}^{(1)}\right)$ as a function of the photon energy at a detection energy of $202.37 \mathrm{eV},(\mathrm{b}) \mathrm{Ar} 2 \mathrm{~s}^{-1}$ photoelectrons at a detection energy of $203.49 \mathrm{eV}$. In this case the "background" contains $\mathrm{Ar} \mathrm{L}_{3} \mathrm{M}_{2,3} \mathrm{M}_{2,3}\left({ }^{1} \mathrm{D}_{2}\right)$ Auger electrons. The solid line indicates a fit without interference. (c) same measurement as in (b) but the solid line represents a fit using a parametrisation containing state-state interference. 
disappear, which is analogous to closing one slit in Young's two-slit experiment. However, the maximum time resolution that can be achieved at present is of the order of $10^{-9} \mathrm{~s}$, which is far too low to actually resolve the time order of the arrival at the detector of the Auger and photoelectron, since the typical life time of an inner-shell vacancy state is of the order $10^{-15} \mathrm{~s}$. Hence, in an actual coincidence experiment the Auger and photoelectron will remain indistinguishable and interferences will therefore still be visible.

\section{On the possibility of a coincidence experiment}

\subsection{Introduction}

Since coincidence experiments are very time consuming, the experiment of van den Brink et al is probably the best candidate to be performed using coincidence techniques. An experiment like this can be done in the experimenter's own laboratory, whereas the photoionisation experiment must be done in a synchrotron radiation laboratory. Beam time on a synchrotron is, in general, scarse and it is not very appealing to try to do a difficult experiment like this in just a few days.

Several experimental groups have succeeded in doing a coincident observation of scattered and ejected electrons in the electron-impact excitation of an autoionising state. Weigold et al [9], Pochat et al [10], Moorhead and Crowe [11], and Lower and Weigold [12] reported with continuously improving energy resolution of the electron analyser $(e, 2 e)$ measurements on the excitation and decay of the autoionising states of helium, at incident energies between 100 and $400 \mathrm{eV}$, and at various scattering and ejection angles.

However, these $(e, 2 e)$ experiments are performed using an electron gun without energy selection, which yields a high current of several $\mu \mathrm{A}$, but a low energy resolution of typically $0.3-0.6 \mathrm{eV}$, whereas the experiment of van den Brink et al was done using an energy-selected electron beam with a low current of $30 \mathrm{nA}$ and a high resolution of $65 \mathrm{meV}$. In the former $(e, 2 e)$ experiments the low resolution is no problem, since it is expected that the cross section does not vary within this resolution, but in an interference experiment like ours it is a problem. To clarify this, consider again figure 1 . If the energy resolution of the incident electrons is low, the electron energy loss is not well defined and the variations in the spectra $1 \mathrm{a}$ to $1 \mathrm{~h}$ will be averaged due to this uncertainty in the energy loss. The question arises whether the interference effects found by van den Brink et al would still be visible.

We have repeated the non-coincidence experiment of van den Brink et al using a low-resolution electron beam to see whether the interference is still observable or not. If our experiment turns out positive it will prove the feasibility of a future coincidence experiment.

\subsection{Experimental results}

We have repeated the experiment of van den Brink et al using a low-resolution ( $300 \mathrm{meV})$, highcurrent $(3 \mu \mathrm{A})$ electron beam. The electrons are measured at a scattering angle of $34^{\circ}$ using a hemi-spherical analyser with an energy resolution of $100 \mathrm{meV}$. The scattering angle is somewhat larger than in the experiments of van den Brink et al $\left(30^{\circ}\right)$, but the present setup does not allow us to choose the same angle. Figure 3 shows the results of two measurements comparable to the results in figure 1. The spectrum in figure $3 \mathrm{a}$ is taken at a constant energy loss that does not correspond to the excitation energy of the $(2 s 2 p)^{1} P$ autoionising state, and should therefore be compared to the spectra in figure $1 \mathrm{a}$ and figure $1 \mathrm{~h}$. The spectrum in figure $3 \mathrm{~b}$ is taken at a constant energy loss that equals the excitation energy of the $(2 s 2 p)^{1} P$ state $(60.15 \mathrm{eV})$, and should therefore be compared with spectrum 1e. Not shown here are a number of these spectra with the constant energy loss given by $60.15+\Delta$, in which $\Delta$ varies stepwise from $-1 \mathrm{eV}$ to $+1 \mathrm{eV}$. In these spectra no systematic changes, like in figure 1, are immediately apparent. Apart from the expected averaging due to the low resolution, this is also due to a non-linear background from which our measurements suffered. 


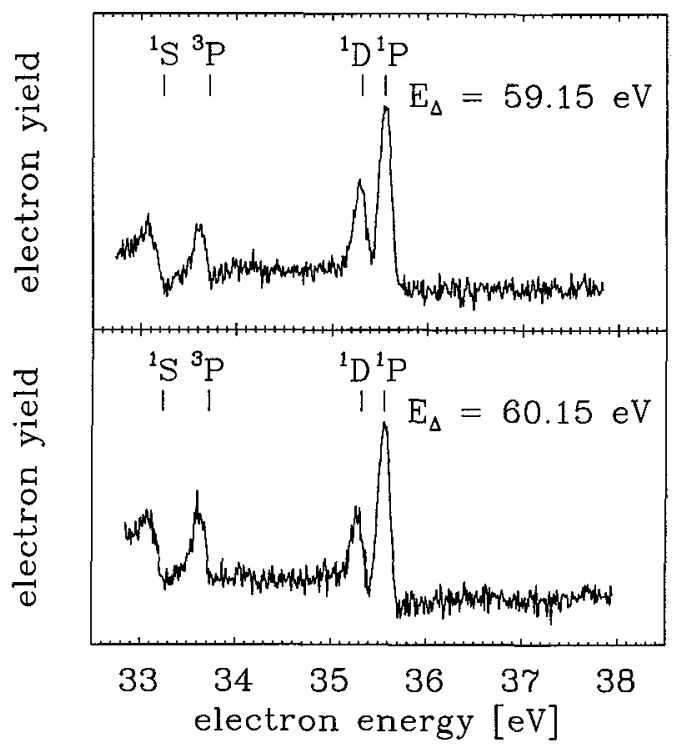

Figure 3: Legend as in figure 1, but these two spectra are measured using a low-resolution high-intensity electron beam.

To see whether there are any systematic changes in the different spectra, we have made fits of Fano profiles to the data. The use of Fano profiles is not completely justified, since the true line shape is given by a convolution between the transmission profile of the electron analyser and the parametrised line shape deduced by van den Brink et al [8], which is able to account for this kind of interference effects. However, the Fano profile is well suited to characterise the data. Interference effects may become visible in any systematic changes in the fit parameters. The Fano profile is given by [13]:

$$
\sigma(\epsilon)=\sigma_{b}+\sigma_{a} \frac{(\epsilon+q)^{2}}{\epsilon^{2}+1}
$$

in which $\sigma_{b}$ is the non-interfering and $\sigma_{a}$ the interfering part of the cross section, $q$ the parameter which determines the shape of the line profile, and $\epsilon$ the reduced energy defined by $\left(E_{i}-E_{r}\right) / \frac{1}{2} \Gamma$ with $E_{i}$ the detection energy, $E_{r}$ the resonance energy and $\Gamma$ the width of the resonance. From equation 2 a few quantities can be deduced. A Fano profile generally consists of a peak and a dip on a background $\sigma_{a}+\sigma_{b}$. The position of the peak is given by $\epsilon=1 / q$ and the cross section at the peak position is $\sigma_{b}+\sigma_{a}\left(q^{2}+1\right)$. This value minus the background is given by $q^{2} \sigma_{a}$, which we will call the peak height. Note that the peak position is not the same as the resonance energy, since a Fano profile is in general an asymmetric line shape. The position of the dip is given by $\epsilon=-q$ and the cross section at the dip position is $\sigma_{b}$.

From the fits we determined the peak positions $(1 / q)$ of the four peaks pertaining to the different autoionising states. In figure 4 the energy differences between the different peaks in one spectrum are given as a function of the constant energy loss. It is clear that sharp structures arise at an energy loss of $60.15 \mathrm{eV}$, which is exactly the excitation energy of the $(2 s 2 p)^{1} P$ state. Apparently, the line shapes of the autoionising states slightly change due to the interference between scattered and ejected electrons. Note again that not the resonance energies have shifted -these stay of course 


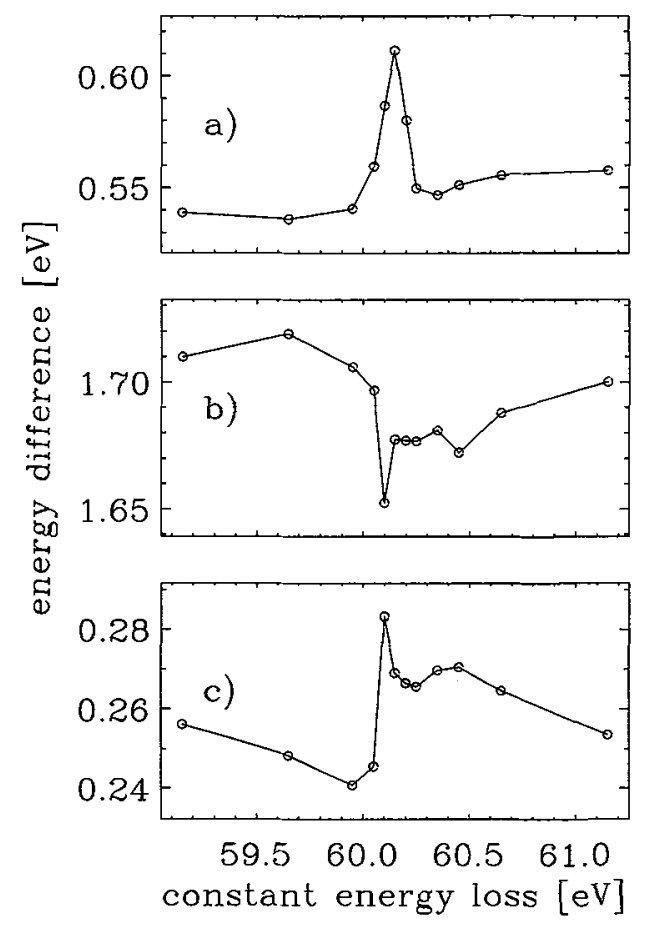

Figure 4: Measured energy differences between the different peaks in one spectrum as a function of the energy loss. (a) gives the difference between the ${ }^{1} S$ and ${ }^{3} P$ peaks, (b) between the ${ }^{3} P$ and ${ }^{1} D$ peaks, and (c) between the ${ }^{1} D$ and ${ }^{1} P$ peaks.

the same- but the shapes of the lines have changed such that the peak positions are shifted.

We also determined from the fits the peak heights of the different peaks $\left(q^{2} \sigma_{a}\right)$. To correct for any non-systematic differences between different measurements, we have normalised the peak heights within one spectrum to the peak height of the $(2 s 2 p)^{1} P$ line. Figure 5 shows the results as a function of the constant energy loss. Again, it is clear that sharp structures arise around the correct energy loss of $60.15 \mathrm{eV}$. Again, due to interference, the shapes of the lines have changed such that the peak heights differ from their normal values.

\subsection{Conclusion}

From both figure 4 and figure 5 we conclude that interference can still be observed despite the low energy resolution of the incident electron beam. However, even more interesting conclusions can be drawn. When comparing figure 5 to figure 1 we see that in figure 5 the $\left(2 s^{2}\right)^{1} S$ peak height has a maximum relative to the $(2 s 2 p)^{1} P$ peak height, whereas in figure 1 the $\left(2 s^{2}\right)^{1} S$ disappears at the energy loss of $60.15 \mathrm{eV}$. Apparently, changing the electron detection direction from $30^{\circ}$ in the case of van den Brink et al to $34^{\circ}$ in our case dramatically changes the interference pattern. This is surprising considering the small change in the scattering angle.

Furthermore, we conclude that a coincident observation of the interference between scattered and ejected electrons seems a possibility for the future. The low-resolution electron beam, required to 


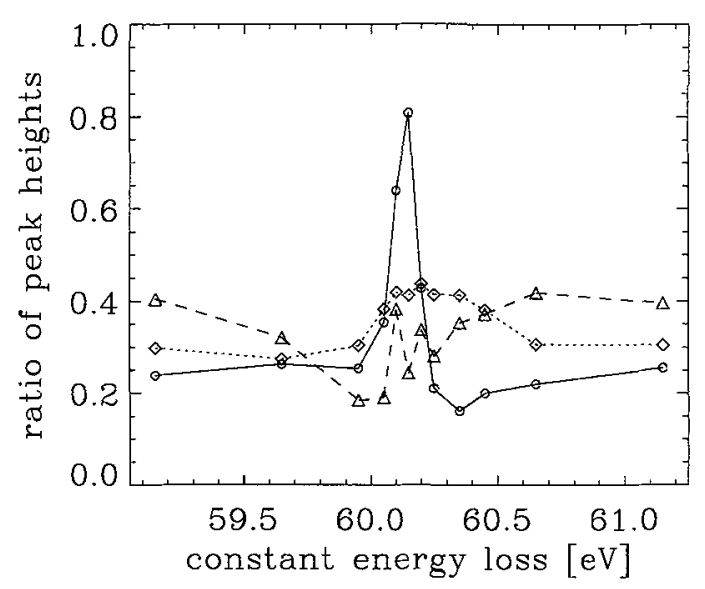

Figure 5: Measured heights of the different peaks, normalised to the height of the ${ }^{1} P$ peak, as a function of the energy loss. Open circles and solid lines represent the height of the ${ }^{1} S$ peak, diamonds and dotted lines the height of the ${ }^{3} P$ peak, and triangles and dashed lines the height of the ${ }^{1} D$ peak.

reach sufficient count rates in an $(e, 2 e)$ experiment, does not totally obscure the interference pattern observed with a high-resolution electron beam.

\section{Acknowledgements}

This work is part of the research program of the 'Stichting voor Fundamenteel Onderzoek der Materie (FOM)', which is financially supported by 'Nederlandse organisatie voor Wetenschappelijk Onderzoek (NWO)'.

\section{References}

[1] Andersen N., Gallagher J.W. and Hertel I.V., Phys. Rep. 165 (1988) 1-188

[2] Andrä H.J., Phys. Scr. 9 (1974) 257-280

[3] Read F.H., J.Phys.B 10 (1977) L207-212

[4] Heideman H.G.M., Coherence and Correlation in Atomic Collisions (Plenum, New York, 1980) pp. $493-508$

[5] Niehaus A., Atomic Inner-Shell Physics (Plenum, New York, 1985) pp. 377-416

[6] van den Brink J.P., van Eck J. and Heideman H.G.M., Phys. Rev. Lett. 61 (1988) 2106-2108

[7] de Gouw J.A., van Eck J., Peters A.C., van der Weg J. and Heideman H.G.M., to be published

[8] van den Brink J.P., Nienhuis G., van Eck J. and Heideman H.G.M., J.Phys.B 22 (1989) 35013518 
[9] Weigold E., Ugbabe A. and Teubner P.J.O., Phys. Rev. Lett. 35 (1975) 209-212

[10] Pochat A., Tweed R.J., Doritch M. and Peresse J., J. Phys, B 15 (1982) 2269-2283

[11] Moorhead P.S.K. and Crowe A., "Excitation of autoionising states of helium in specific momentum transfer electron collisions", Int. Conf. on Physics of Electronic and Atomic Collisions, Palo Alto 24-30 July 1985 (Palo Alto, 1985) p. 160

[12] Lower J. and Weigold E., J. Phys. B 23 (1990) 2819-2845

[13] Fano U., Phys. Rev. 124 (1961) 1866-1878 